\title{
Rectangular jet with shear layer swirl: Rotation \& mixing enhancement
}

\section{Saeed Farokhi and Ray R Taghavi}

@SAGE

\begin{abstract}
A rectangular jet emerging from a nozzle with embedded swirl vanes in its exit boundary layer is studied. The swirling shear layer imposes an external torque on the jet boundary where it causes jet rotation in the direction of axis switching, skew deformation as well as enhanced mixing. In moderate aspect ratio rectangular nozzles, e.g. $A R=5:$, a pair of co-rotating streamwise vortices is formed on the narrow boundary of the jet that dominates its dynamics in the near field. A vortex-induced model is developed that accounts for the rotation of the rectangular jet with embedded shear layer swirl. The model also shows that the jet rotation is diminished with increasing aspect ratio, as $\mathrm{AR}^{-2}$. The higher entrainment rate in the rotating jet with skew deformation causes the jet mass flow rate with shear layer swirl to be $20-25 \%$ higher than the corresponding plain rectangular nozzle of the same aspect ratio $\left(x / D_{e}>1\right)$. The proposed model is validated using computational simulation results of previous investigations that appeared in the literature.
\end{abstract}

\section{Keywords}

rectangular jet, swirl, shear layer with swirl, mixing enhancement, axis switching

Date received: I4 March 20I5; revised: 21 June 20I5; accepted: 2 July 2015

\section{Introduction}

Turbulent-free jets, with and without swirl and excitation, have been the subject of extensive studies at NASA under the leadership and collaboration with Rice. ${ }^{1-8}$ Vortex roll up in the emerging shear layer and the subsequent large-scale structures coupled with forced excitations, i.e. instability waves, to create mixing enhancement in turbulent free jets. Asymmetric jets, e.g. emerging from elliptic or rectangular nozzles, created new dynamics known as axis switching. Hussain and Husain ${ }^{9}$ and Zaman $^{10}$ among other researchers have experimentally studied this phenomenon. The induced dynamics of azimuthal vorticity, $\omega_{\theta}$ in asymmetrical jets is the dominant mechanism responsible for the phenomenon of axis switching.

Aerospace Engineering Department, The University of Kansas, Lawrence, KS, USA

\section{Corresponding author:}

Saeed Farokhi, Aerospace Engineering Department, The University of Kansas, Lawrence 66045, KS, USA.

Email: sfarokhi@ku.edu 
However, streamwise vorticity, $\omega_{\mathrm{x}}$-induced dynamics, also contributes to the evolution of an asymmetric jet, as noted by Zaman ${ }^{10}$ and Samimy et al. ${ }^{11}$ These two dynamics are coupled and compete with each other, which may hinder or promote switchover of asymmetric jets. The study of coupled interaction of $\omega_{\theta}-\omega_{\mathrm{x}}$ using delta tabs, as a means of generating streamwise vorticity, is experimentally conducted. ${ }^{11-14}$ Zaman et al. ${ }^{15}$ recently wrote an excellent review of this topic. The role of swirl in free turbulent jets is to trigger centrifugal instability, set up radial pressure gradient and thus promote mixing. ${ }^{8,16}$ However, swirl in aircraft exhaust systems creates a penalty on thrust production, ${ }^{17}$ which limits its deployment to very selective applications, e.g. in combustion chambers. It is important to reiterate that the phenomenon of "axis switching" driven by $\omega_{\theta}$ and $\omega_{\mathrm{x}}$ dynamics in elliptical or rectangular jets do not involve physical rotation of the jet, rather the shrinkage in one dimension and the elongation in another that may repeat in the streamwise direction. However, when a jet is subjected to external torque through induced swirl in its shear layer, as in the present study, the phenomenon of "axis switching" involves the physical jet rotation. In this sense, it is a new dynamics in turbulent jets that create "axis switching".

To minimize the thrust penalty of large-scale (bulk) swirling jets, the case of embedded swirl in the nozzle exit boundary layer is investigated. ${ }^{18,19}$ The solution offers the benefits of swirl in mixing enhancement without a major penalty of thrust loss. The external torque in our configuration, as an initial boundary condition, dominates the mechanism of jet rotation, whereas the switchover phenomenon in asymmetric jets is caused by $\omega_{\theta}$-dynamics. We proceed to model the former mechanism.

\section{Rectangular jet with swirling shear layer}

A 5:1 aspect ratio rectangular nozzle has short and long (inner) dimensions of 6 and $30 \mathrm{~mm}$, respectively. The equivalent round diameter of the jet is $D_{e}=15.1 \mathrm{~mm}$. The nozzle exit has incorporated counterclockwise vanes, with vane angle of $\theta_{0}=30^{\circ}$ with respect to the nozzle's axial direction. The vanes are carried over to the sides of the nozzle as well. The height of the exit swirl vanes is $1 \mathrm{~mm}$. The initial swirl distribution in the shear layer is assumed to be $\mathrm{V}_{\mathrm{x}} \tan \theta_{0}$, where $\mathrm{V}_{\mathrm{x}}$ is the initial axial velocity.

The nozzle is of convergent type and is under-expanded with choked exit. The jet Mach number, corresponding to perfect expansion, is 1.53.

The temperature at the nozzle exit is $250 \mathrm{~K}$ and the fluid density is $1.414 \mathrm{~kg} / \mathrm{m}^{3}$. Additional description for initial condition, boundary conditions and CFD parameters is detailed in Han et al. ${ }^{18,19}$

Schematic drawing of the rectangular nozzle with its exit flow condition is shown in Figure 1(a). Figure 1(b) shows the counterclockwise vane placement and its geometry in the nozzle exit. The flow in the $\mathrm{y}-\mathrm{z}$ plane shows that the boundary layer swirl is confined to a narrow strip around the periphery and is in counter-clockwise direction. Figure 2 shows the formation of two corner vortices on the short dimensions of the nozzle that are co-rotating and are in streamwise direction. The diameter of these corner vortices in moderate to high aspect ratio nozzles, say $>5: 1$, scales as $h$.

\section{The dynamics of the jet in near field}

The external torque that was applied to the rectangular jet at the nozzle exit manifests itself in angular momentum distribution along the broad and short dimensions of the jet. 


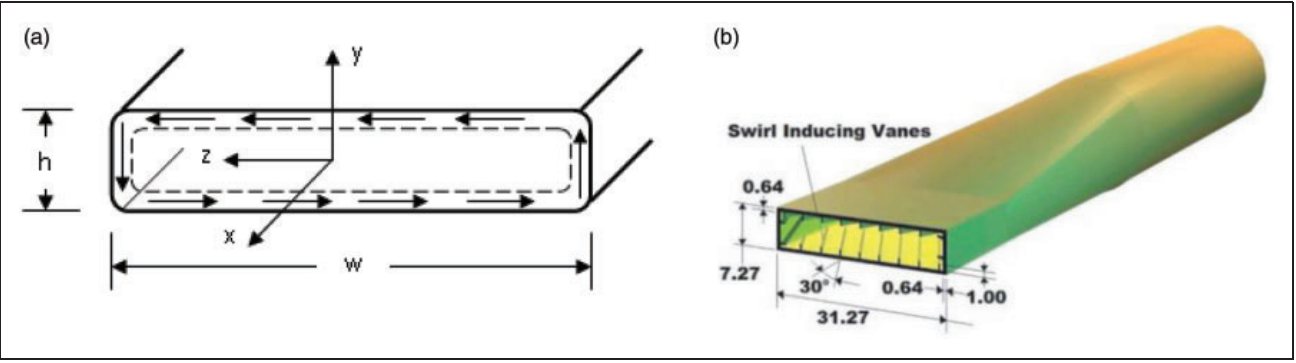

Figure I. (a) Definition sketch of the rectangular nozzle exit geometry with embedded boundary layer swirl and coordinate system. (b) Details of the counterclockwise swirl vanes and their dimensions in the nozzle exit (from Han et al. ${ }^{19}$ ).

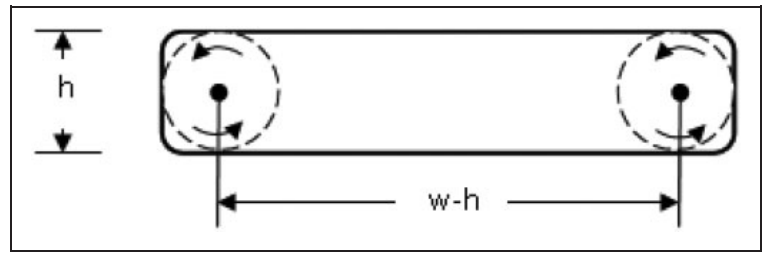

Figure 2. Corner flowfield modeled as a pair of co-rotating vortex filaments in high-aspect ratio rectangular nozzle.

The geometrically imposed boundary condition on the narrow side creates a pair of streamwise vortices that scale as $\mathrm{h}$ and with centerline distance of $\mathrm{w}-\mathrm{h}$, as shown in Figure 3 . These vortices act as a pair and rotate in the counterclockwise direction, thus controlling the dynamics of jet rotation in the near field.

The angular momentum distribution on the broad side of the jet has a small moment arm from the $z$-axis, as shown in Figure 4. A representative distribution of the shear in the cross plane, $\mathrm{y}-\mathrm{z}$, is depicted in Figure 4, that shows a plane of symmetry. The role of the broad side shear distribution on the near field jet dynamics is to cause a skew in the rectangular jet, as depicted in Figure 5.

\section{Jet rotation model}

The initial swirl induced in the nozzle exit boundary layer is

$$
\left.V_{\theta 0}\right]_{\text {Shear Layer }}=V_{x} \sin \theta_{0}
$$

The corner vortex circulation is

$$
\Gamma=\pi h V_{\theta 0}=\pi h V_{x} \sin \theta_{0}
$$

The self-induced rotational speed of the jet is

$$
V_{\theta}=\frac{\Gamma}{2 \pi(w-h)}=\frac{1}{2}\left(\frac{h}{(w-h)}\right) V_{x} \sin \theta_{0}
$$




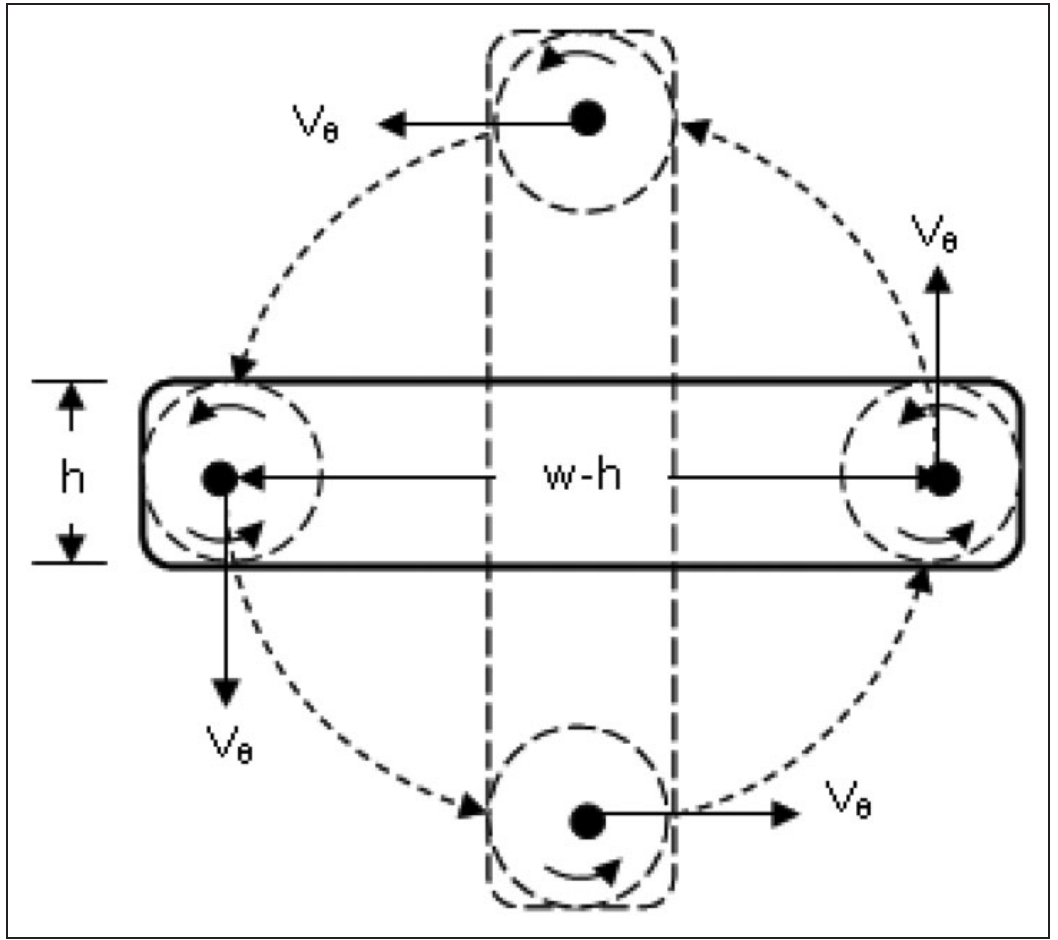

Figure 3. Circular trajectory of a pair of co-rotating vortices in the corner regions of the rectangular jet.

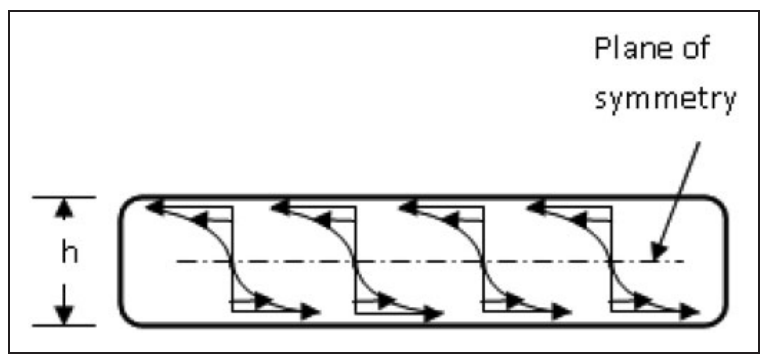

Figure 4. The vortex sheet model along the broad sides of the rectangular jet shear layer with embedded swirl.

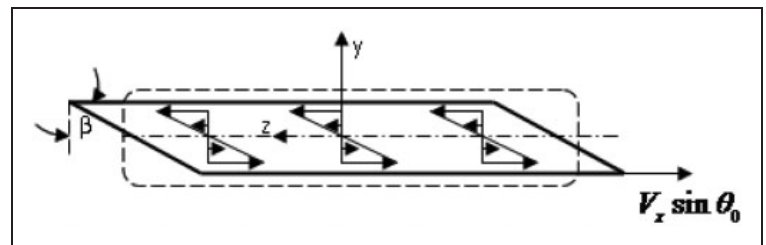

Figure 5. The rectangular jet skew deformation caused by the broad side shear due to embedded swirl. 
The ratio of rotational-to-axial speed is thus

$$
\frac{V_{\theta}}{V_{x}}=\frac{1}{2}\left(\frac{h}{(w-h)}\right) \sin \theta_{0}
$$

The nozzle aspect ratio, AR, is defined as

$$
A R \equiv \frac{w}{h}
$$

Thus, the ratio of rotational-to-axial speed in the jet, in terms of the nozzle aspect ratio, is

$$
\frac{V_{\theta}}{V_{x}}=\frac{1}{2}\left(\frac{1}{A R-1}\right) \sin \theta_{0}
$$

The period of rotation is approximately

$$
T \approx \frac{\pi w}{V_{\theta}}=\frac{2 \pi w}{V_{x} \sin \theta_{0}}(A R-1) \quad \theta_{0} \neq 0
$$

Axis switching phenomenon, in the context of the present problem, corresponds to $\mathrm{T} / 4$, therefore

$$
\frac{T}{4}=\frac{\pi w}{2 V_{x} \sin \theta_{0}}(A R-1) \quad \theta_{0} \neq 0
$$

The streamwise position along the jet axis where axis-switching takes place is

$$
x_{\text {axis-switching }}=\frac{V_{x} T}{4}=\frac{\pi w}{2 \sin \theta_{0}}(A R-1) \quad \theta_{0} \neq 0
$$

Dividing equation (8a) by the small dimension of the nozzle, and replacing $\mathrm{w} / \mathrm{h}$ by aspect ratio, we get the non-dimensional distance along the jet where axis switching takes place, namely

$$
\frac{x_{\text {axis-switching }}}{h}=\left(\frac{\pi}{2 \sin \theta_{0}}\right) A R(A R-1) \quad \theta_{0} \neq 0
$$

Since equation ( $8 \mathrm{~b}$ ) shows that the location of switchover, by initial torque alone, scales as $A R^{2}$, the effect of jet rotation is diminished with $\mathrm{AR}^{-2}$. We can also write equation (8b) in terms of rotation angle, $\theta$, as

$$
\frac{x(\theta)}{h}=\left(\frac{\pi}{2 \sin \theta_{0}}\right) A R(A R-1)\left(\frac{\theta}{90}\right) \quad \theta_{0} \neq 0
$$

where $\theta$ is in degrees.

\section{The jet skew deformation}

To estimate the initial phases of the jet skew deformation corresponding to the near field, we approximate the shear stress on the broad side of the jet. Shear stress $\tau_{\mathrm{yz}}$ is proportional to azimuthal velocity gradient and in a rectangular nozzle it exhibits a plane of symmetry. The first-order approximation of the shear is based on the linear velocity distribution between the 
upper and lower shear layers on the broad side. Therefore

$$
\tau_{y z} \approx\left(\mu+\mu_{t}\right)\left(\frac{V_{x} \sin \theta_{0}}{h / 2}\right)
$$

The rate of strain deformation in $\mathrm{y}-\mathrm{z}$ plane, $\varepsilon_{\mathrm{yz}}$, for a Newtonian fluid is therefore approximated by

$$
\frac{d B}{d t} \approx \frac{V_{x} \sin \theta_{0}}{h / 2}
$$

The conclusion from this simple model on the rate of deformation, or skew, in $\mathrm{y}-\mathrm{z}$ plane, i.e. the jet cross sectional plane, is that shear deformation is proportional to the sine of the initial swirl angle, $\theta_{0}$, and inversely proportional to the narrow dimension of the jet, $\mathrm{h}$. In the vanishing limit of the narrow dimension of the jet, the jet skew deformation becomes large and dominant. Therefore, a high aspect ratio "slit" nozzle, as found in low-observable military aircraft, with boundary layer swirl can be modeled as a thin vortex sheet where the jet dynamics is purely driven by the self-induced vortex sheet rollup, as outlined in Batchelor. $^{20}$

\section{Model validation}

To study the characteristics of supersonic rectangular jets, a computational code known as the three-dimensional Proteus code ${ }^{21-23}$ was used with a shock-adaptive grid generator developed by Han et al. ${ }^{18,19}$ The turbulence models used in the calculations were BaldwinLomax and Chien $\mathrm{k}-\varepsilon$ turbulence models. Chien $\mathrm{k}-\varepsilon$ turbulence model was applied to the computation after the results were obtained by using Baldwin-Lomax turbulence model in the first iteration. Han et al. ${ }^{18,19}$ describe in detail the computational simulation approach from grid generation, shock-adaptive scheme, convergence rate and numerical stability considerations. For the sake of brevity, these will not be reproduced here.

Figure 6 shows a side-by-side comparison of the axial velocity contours in the cross plane at three axial locations of a rectangular jet with embedded shear layer swirl (on the left) and one without swirl (on the right). The jet subjected to initial torque rotates in the direction of switchover in addition to undergoing skew deformation. The jet rotation based on the proposed model, i.e. equation $(8(\mathrm{c}))$, at $\mathrm{x} / \mathrm{h}=7.5$ predicts $10.7^{\circ}$ and the rotation at $\mathrm{x} / \mathrm{h}=11.5$ is predicted to be $16.4^{\circ}$. These rotation angles are drawn as straight lines (to scale) in Figure 6(c) and (e) for the corresponding axial positions. For the purpose of model validation, these lines reasonably represent the jet rotation in the near field. In terms of jet equivalent diameter, $D_{e}$, the two axial positions are at 3.0 and 4.6 , respectively.

The effect of embedded swirl on jet entrainment and thus mass flow rate is shown in Figure 7 . The computational results indicate between 20 and $25 \%$ mass flow rate increase in the jet with shear layer swirl (at $\mathrm{x} / \mathrm{D}_{\mathrm{e}}=1.0$ and $\mathrm{x} / \mathrm{D}_{\mathrm{e}}=5.0$, respectively) over its swirl-free counterpart.

\section{Summary and conclusions}

A turbulent rectangular jet subject to initial shear layer swirl is studied. A near-field model, based on vorticity distribution, is developed that predicts the jet rotation and skew deformation, which identifies a new axis-switching phenomenon in non-axisymmetric jets. 


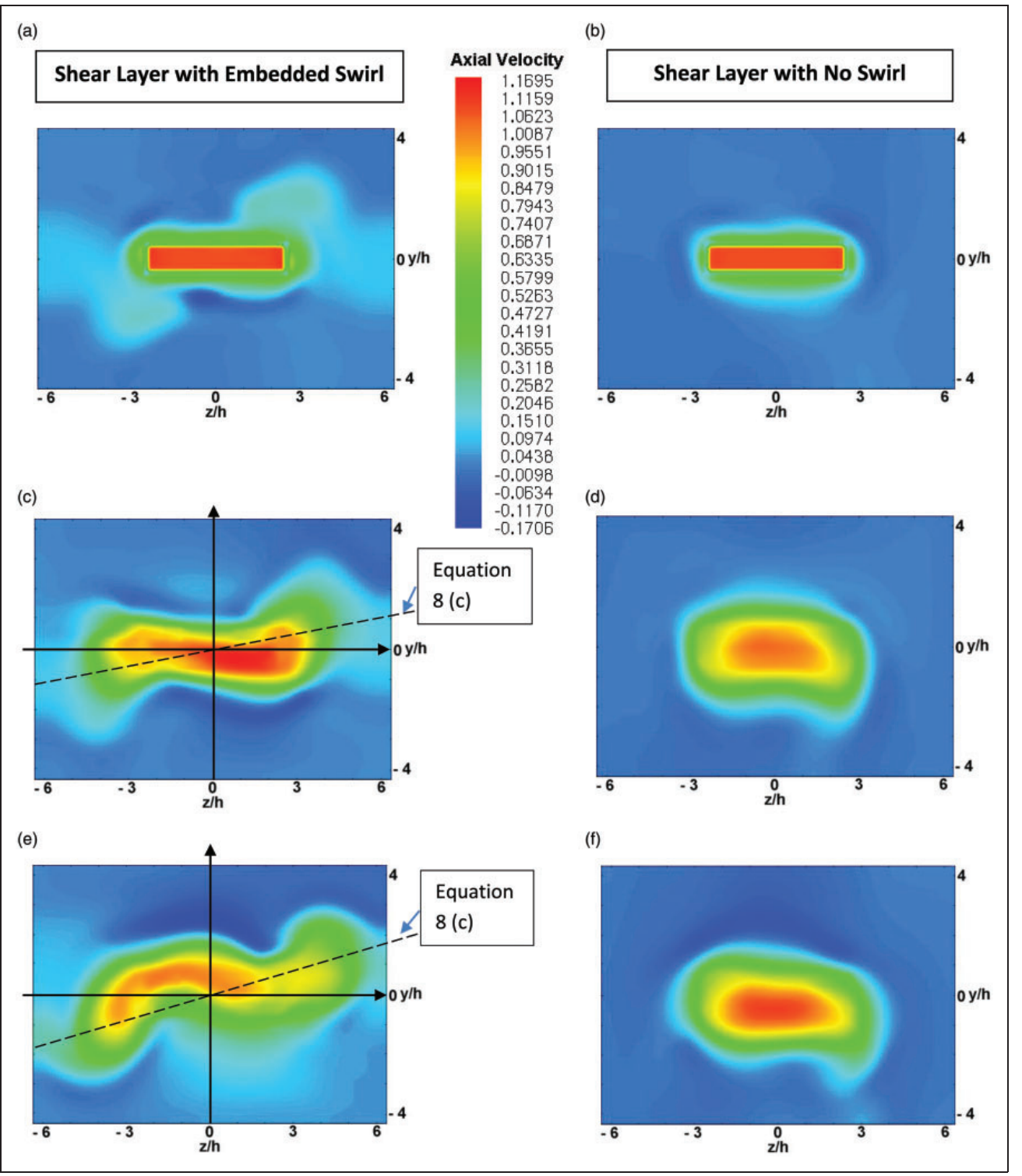

Figure 6. Axial velocity contour plots at $\mathrm{x} / \mathrm{h}=0,7.5$ and II .5 downstream of a rectangular nozzle with and without boundary layer swirl (the dashed lines in parts (c) and (e) correspond to the jet rotation model, equation 8(c)).

The physical jet rotation model in the $x$-direction, i.e. along the jet axis, is compared to 3-D computational (RANS) simulation, which seems to be in substantial agreement. The model suggests diminished jet rotation with increasing aspect ratio in proportion to $\mathrm{AR}^{-2}$. The embedded swirl in the nozzle boundary layer adversely impacts thrust production, but the enhanced entrainment (20-25\%) and thus jet spread proves useful in the design of advanced low-observable aircraft. 


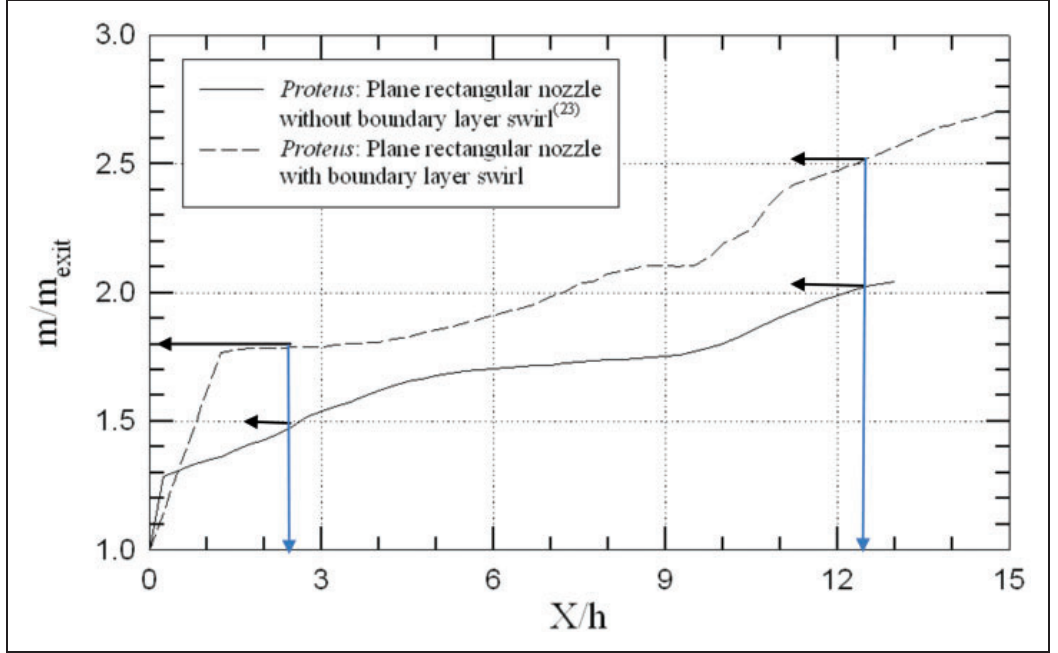

Figure 7. Comparison of mass flow rates for the plane rectangular nozzle with and without boundary layer swirl in the near field (the arrows correspond to $X / D e=1.0$ and 5.0) (data extracted from Han et al. ${ }^{18}$ ).

\section{Acknowledgement}

The authors are indebted to Edward J Rice for his guidance, unwavering support and friendship. We learned about our own research through discussions with Ed Rice, Khairul Zaman, Ganesh Raman, Reda Mankbadi, J Panda among many others who made CW-17 the most productive Lab at NASALeRC. The efforts of Mr Ralph Fallert, who provided technical support to the research team for many years with modifications, nozzle installations, and component fabrications in CW-17 and other research facilities at NASA LeRC are also greatly appreciated. We are indebted to all of our graduate students, but in particular, to our doctoral students, Gary Cheng, Charlie Wu, Ron Barrett, Kyle Wetzel, Wonjoong Lee, Sueng-Jae Hwang, SY Han, Wonjin Jin, Leslie Smith, Sonja Flesberg, Alex Karwas, and Amool Raina, who have been our partners in research.

\section{Declaration of conflicting interests}

The author(s) declared no potential conflicts of interest with respect to the research, authorship, and/or publication of this article.

\section{Funding}

The author(s) received no financial support for the research, authorship, and/or publication of this article.

\section{References}

1. Taghavi RR, Rice EJ and Farokhi S. Controlled excitation of a cold turbulent swirling free jet. Transact ASME J Vibrat Acoust Stress Reliab Des 1988; 110: 234-237.

2. Farokhi S, Taghavi RR and Rice EJ. Effect of initial swirl distribution on the mean evolution of a turbulent jet. AIAA J 1989; 27: 700-706. 
3. Taghavi RR, Farokhi S and Rice EJ. Large-amplitude acoustic excitation of swirling turbulent jets. In: AIAA second shear flow control conference, Tempe, AZ, March 1989. AIAA Paper 89-0970.

4. Farokhi S, Taghavi R and Rice EJ. Modern developments in shear flow control with swirl. AIAA J 1992; 30: 1482-1483.

5. Rice EJ and Raman G. Enhanced mixing of a rectangular supersonic jet by natural and induced screech. AIAA-93-3263, Presented at AIAA Shear Flow Conference, Orlando, FL, 1993.

6. Wu C, Farokhi S and Taghavi R. On spatial instability of a swirling jet: theory and experiment. AIAA J 1992; 30: 1545-1552.

7. Raman $\mathrm{G}$ and Rice EJ. Instability modes excited by natural screech tones in a supersonic rectangular jet. Phys Fluid 1994; 6: 3999-4008.

8. Farokhi S and Taghavi RR. Mixing enhancement in a jet with arbitrary swirl. In: Proceedings of fifth international conference on computational methods and experimental measurements, Montreal, Canada, 23-26 July 1991.

9. Hussain F and Husain HS. Elliptic Jets, Part 1: characteristics of unexcited and excited jets. $J$ Fluid Mech 1989; 208: 257-320.

10. Zaman KMBQ. Effect of delta tabs on mixing and axis switching in jets from asymmetric nozzles. NASA-TM 106450, Presented at the 32nd Aerospace Sciences Meeting, Reno, NV, 1994.

11. Samimy M, Zaman KMBQ and Reeder MF. Effect of tabs at the nozzle lip on the flow and noise field of an axisymmetric jet. AIAA J 1993; 31: 609.

12. Ahuja KK and Brown WH. Shear flow control by mechanical tabs. AIAA Paper 89-0994, Paper presented at the 2nd Shear Flow Conference, Tempe, AZ, 1989.

13. Zaman KMBQ, Reeder MF and Samimy M. Control of an axisymmetric jet using vortex generators. Phys Fluids A 1994; 6: 778.

14. Bradbury LJS and Khadem AH. The distortion of a jet by tabs. J Fluid Mech 1975; 20: 801.

15. Zaman KMBQ, Bridges JE and Huff DL. Evolution from 'tabs' to 'chevron' technology - a review. In: Raman G, Srinivasan K (eds) Frontiers in aeroacoustics. Essex, UK: Multi-Science Publishing Co. Ltd, 2014.

16. Panda $\mathbf{J}$ and McLaughlin DK. Experiments on the instabilities of a swirling jet. Phys Fluid 1994; 6: 263-276.

17. Farokhi S. Aircraft propulsion, 2nd ed. Chichester, UK: John Wiley and Sons, Ltd., 2014.

18. Han SY, Taghavi RR and Farokhi S. Passive control of supersonic rectangular jets through boundary layer swirl. Int J Turbo Jet-Engine 2013; 30: 199-216.

19. Han SY, Taghavi RR and Farokhi S. Numerical simulation of supersonic jet instability modes of plane and notched rectangular nozzles. Proc IMechE, Part C: J Mechanical Engineering Science 2012; 226: 105-122.

20. Batchelor GK. An introduction to fluid dynamics. Cambridge, UK: Cambridge University Press, 1967.

21. Towne CE, Schwab JR and Bui TT. Proteus three-dimensional Navier-Stokes computer code Version 1.0. Vol. 1 - Analysis Description, NASA TM-106337, Document ID: 19940012054, 01 October 1993.

22. Towne CE, Schwab JR and Bui TT. Proteus three-dimensional Navier-Stokes computer code Version 1.0; Vol. 2 - User's Guide. NASA TM-106340, Document ID: 19940011015, 01 October 1993.

23. Towne CE, Schwab JR and Bui TT. Proteus three-dimensional Navier-Stokes computer code Version 1.0; Vol. 3 - Programmer's Reference, NASA TM-106341, Document ID: 19940011392, 01 October 1993. 
Copyright of International Journal of Aeroacoustics is the property of Sage Publications Inc. and its content may not be copied or emailed to multiple sites or posted to a listserv without the copyright holder's express written permission. However, users may print, download, or email articles for individual use. 